\title{
Anisotropic propagation of Galactic cosmic-rays and spectral hardening in the Galactic Center
}

\author{
Andrea Vittino* \\ Physik-Department T30d, Technische Universität München, James Franck-Str. 1, D-85748, \\ Garching, Germany \\ E-mail: andrea.vittino@tum.de
}

\section{Silvio Sergio Cerri}

Dipartimento di Fisica “E. Fermi”, Università di Pisa, Largo B. Pontecorvo 3, I-56127 Pisa, Italy

E-mail: silvio.cerriedf.unipi.it

\section{Daniele Gaggero}

GRAPPA, University of Amsterdam, Science Park 904, 1098 XH Amsterdam, Netherlands

E-mail: d.gaggero@uva.nl

\section{Carmelo Evoli}

Gran Sasso Science Institute, Viale Francesco Crispi 7, 67100 L'Aquila, Italy

E-mail: carmelo.evoli@gssi.it

\section{Dario Grasso}

INFN Pisa, Largo B. Pontecorvo 3, I-56127 Pisa, Italy

E-mail: dario.grasso@pi.infn.it

\begin{abstract}
Spatial diffusion under the influence of the Galactic magnetic field (GMF) plays a crucial role in the transport of cosmic-ray protons. The standard assumption is that the GMF is purely azimuthal and the diffusion process can be considered to be uniform and isotropic all across the Galaxy. However, this picture might be inaccurate, since both theoretical arguments and numerical simulations predict that diffusion might exhibit different features, depending on its being parallel or perpendicular to the direction of the magnetic field. This might have a large impact in the Galactic Center region, where the GMF presents a strong out-of-plane component. In particular, an anisotropic diffusion could contribute in determining the non-uniform density and energy distribution of cosmic-ray protons that is inferred from the measurements of the gamma-ray emissivity along the Galactic plane. Here we discuss how anisotropic cosmic-ray diffusion can be implemented within a realistic model of cosmic-rays transport. In particular, we investigate how the various features in the modelling of the GMF can impact the spatial and energetic distribution of cosmic-ray protons and we illustrate how this can be related to the most recent gamma-ray observations.
\end{abstract}

35th International Cosmic Ray Conference - ICRC2017

10-20 July, 2017

Bexco, Busan, Korea

${ }^{*}$ Speaker. 


\section{Introduction}

In the standard picture of Galactic cosmic-ray (CR) transport, the process of spatial diffusion is usually assumed to be uniform and isotropic. It is typically described in terms of a diffusion coefficient whose dependence on the CR rigidity is in the form of a spatially independent powerlaw scaling: $D \propto(p / Z)^{\delta}$ [1]. For decades, models of CR propagation based on this assumption have been successfully adopted to reproduce a vast plethora of experimental data [2]. However, both theoretical arguments and anomalies reported in recent observations may have started to cast some doubts over this simple scenario and might be calling for a more complex modelling of $\mathrm{CR}$ diffusion.

On the experimental side, important information comes from non-local observables, especially in the gamma-ray channel. In particular, the gamma-ray flux measured by Fermi-LAT seems to hint at a CR proton slope that hardens in the inner Galaxy, as shown in [3, 4]. A possible interpretation of such a hardening is in terms of an evolution of the spatial diffusion rigidity scaling with the Galactocentric radius $R, D \propto(p / Z)^{\delta(R)}$ [5]. Models featuring a spatial diffusion of this kind have been shown in $[6,7]$ to reproduce not only Fermi-LAT data, but also measurements reported by H.E.S.S. and Milagro.

On the theoretical side, a highly anisotropic transport regime is predicted by the Quasi-Linear Theory (QLT) of pitch-angle scattering $[8,9,10]$, which is expected to hold when dealing with the Galactic transport of CRs with energy in the GeV-TeV range [11]. If one denotes with $D_{\|}$and $D_{\perp}$ the diffusion coefficients along and perpendicular to the direction of the regular component of the Galactic Magnetic Field (GMF), the QLT predicts:

$$
\frac{D_{\perp}}{D_{\|}} \sim \mathscr{F}(k) \sim \frac{\delta B_{k}^{2}}{B_{0}^{2}} \ll 1 .
$$

Moreover, numerical simulations involving high-energy CRs predict a different rigidity scaling for $D_{\|}$and $D_{\perp}[12,11,13]$. Lastly, recent investigations on the topic of Galaxy formation show that a magnetic field amplification compatible with current observations requires CR transport to be anisotropic [14].

Given these premises, we present here a numerical implementation of anisotropic diffusion in the framework of the DRAGON code $^{1}$ [15]. This proceeding, which is based on [16], is organized as follows: In Section 2 we describe the setup of our model, while in Section 3 we present our results, first in the framework of a toy model and then in connection with gamma-ray observations. We then report our conclusions in Section 4.

\section{Our setup}

We adopt a numerical solver specifically built to deal with a fully anisotropic CR transport equation. This solver is implemented in the framework of the DRAGON code and will be adopted also in its new version, DRAGON2 [17]. We address the reader to the Appendix of [16] for a more detailed discussion. Here we describe its main features ${ }^{2}$.

\footnotetext{
${ }^{1}$ www.dragonproject.org

${ }^{2}$ An alternative approach to model anisotropic diffusion, based on a stochastic differential equation technique, can be found in [18].
} 


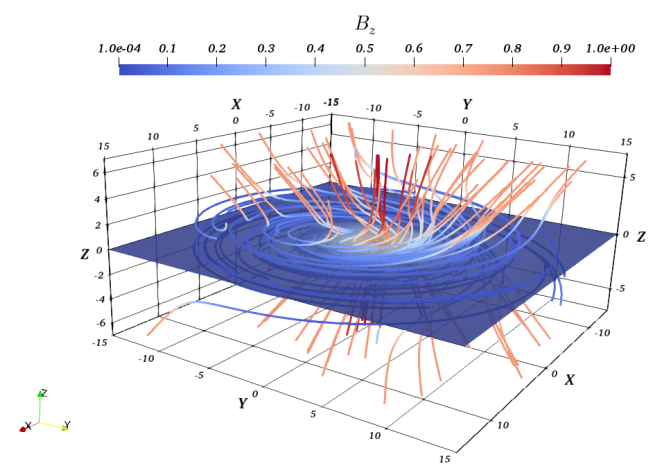

Figure 1: Three-dimensional representation of the realistic GMF model used in our simulations and defined by Eqs. (2.7)-(2.13). The values of $B_{z}$ is shown with colors on top of the magnetic field lines and as a contour plot on the $z=0$ Galactic plane.

For the purposes of this work, we consider a simplified scenario where only the ingredients that are relevant to describe the transport of high energy protons are taken into account. This means that processes such as advection, energy losses and reacceleration are neglected. Under such assumption, the $\mathrm{CR}$ transport equation can be written as:

$$
\frac{\partial N}{\partial t}=\nabla \cdot(\mathbf{D} \cdot \nabla N)+S=\frac{\partial}{\partial x_{i}}\left(D_{i j} \frac{\partial N}{\partial x_{j}}\right)+S,
$$

where $N$ denotes the CR density, while $S$ represents the source term and $\mathbf{D}$ is the diffusion tensor.

We restrict ourselves to the two-dimensional case, which means that we work under the assumption of azimuthal symmetry and CRs are assumed to diffuse in a cylinder in which we define a coordinate system $(R, z)$, with radius $R \in\left[0, R_{\max }\right]$ and $z \in[-H,+H]$. The spatial grid on which Eq. (2.1) is discretised has a resolution of $0.1 \mathrm{kpc}$ in both the $R$ and $z$ directions.

The source term $S$ is modelled according to the parametrization based on pulsar catalogs introduced in [19], while the components of the diffusion tensor $D_{i j}$ are defined as:

$$
D_{i j} \equiv D_{\perp} \delta_{i j}+\left(D_{\|}-D_{\perp}\right) b_{i} b_{j}, \quad b_{i} \equiv \frac{B_{i}}{|\mathbf{B}|},
$$

with $\mathbf{B}$ being the ordered magnetic field, while $\mathbf{b}=\mathbf{B} /|\mathbf{B}|$ is its unit vector. The quantities $D_{\|}$and $D_{\perp}$ represent the diffusion coefficients for the CR transport in a direction parallel and perpendicular to the direction of the GMF, respectively. Both these coefficients are assumed to be spatially homogeneous, but their rigidity scaling and their normalizations are different:

$$
D_{\|}=D_{0 \|}\left(\frac{p}{Z}\right)^{\delta_{\|}} \quad \text { and } \quad D_{\perp}=D_{0 \perp}\left(\frac{p}{Z}\right)^{\delta_{\perp}} \equiv \varepsilon_{D} D_{0 \|}\left(\frac{p}{Z}\right)^{\delta_{\perp}}
$$

In this work we cosider the cases $\delta_{\|}=0.3$ and $\delta_{\|}=0.1$ (the latter only for toy models, as it will be discussed in the following), while $\varepsilon_{D} \in[0.01,1]$ and $\delta_{\perp} \in[0.5,0.7]$ in agreement with a low-energy extrapolation of the numerical simulations conducted in $[12,11,13]$. It is important to remark that, as one can easily see from eqs. (2.2) and (2.3), even if $D_{\|}$and $D_{\perp}$ are assumed to be uniform, the 
global diffusion coefficient $D$ exhibits a spatial dependence, that is related to the geometry of the GMF. As for the GMF, we consider two models:

- A simplified toy model characterised by:

$$
\begin{aligned}
& B_{r}=0 \\
& B_{\phi}=B_{0, \phi}\left(1-e^{-r / R_{0}}\right) \\
& B_{z}=B_{0, z} e^{-r / R_{0}} \equiv \varepsilon_{B} B_{0, \phi} e^{-r / R_{0}} .
\end{aligned}
$$

- A more realistic model, shown in Fig. 1, based on the parametrisations defined in [20, 21], where the GMF is the sum of three components: a disk, a halo and a poloidal field, labelled as $B_{\phi}^{\text {disk }}, B_{\phi}^{\text {halo }}$ and $B_{z, R}^{\text {pol }}$, respectively. The disk and halo components are taken to be purely azimuthal:

$$
\begin{gathered}
B_{\phi}^{\text {disk }}(R, z)=\left\{\begin{array}{cc}
B_{D 0} e^{-|z| / z_{0}} & \left(R<R_{C D}\right) \\
B_{D 0} e^{-|z| / z_{0}} e^{-\left(R-R_{\circ}\right) / R_{0}} & \left(R>R_{C D}\right)
\end{array}\right. \\
B_{\phi}^{\text {halo }}(R, z)=B_{H 0}\left[1+\left(\frac{|z|-z_{0}^{H}}{z_{1}^{H}}\right)\right]^{-1} \frac{R}{R_{O}^{H}} e^{\left(1-\frac{R}{R_{0}^{H}}\right)}
\end{gathered}
$$

while the poloidal field, which follows the model presented in [21] is confined in the $(R, z)$ plane and it is characterised by an $\mathrm{X}$-shape parametrised as:

$$
\begin{gathered}
B_{z}^{\mathrm{pol}}(R, z)=B_{\mathrm{X}}(R, z) \cos \left[\Theta_{\mathrm{X}}(R, z)\right], \\
B_{R}^{\mathrm{pol}}(R, z)=B_{\mathrm{X}}(R, z) \sin \left[\Theta_{\mathrm{X}}(R, z)\right],
\end{gathered}
$$

where $B_{\mathrm{X}}$ and $\Theta_{\mathrm{X}}$ are defined as

$$
\begin{gathered}
B_{\mathrm{X}}(R, z)=\left\{\begin{array}{cc}
B_{\mathrm{X}}^{0}\left(\frac{R_{p}}{R}\right)^{2} e^{-R_{p} / R_{\mathrm{X}}} & \left(R \leq R_{\mathrm{X}}^{c}\right) \\
B_{\mathrm{X}}^{0}\left(\frac{R_{p}}{R}\right) e^{-R_{p} / R_{\mathrm{X}}} & \left(R>R_{\mathrm{X}}^{c}\right)
\end{array}\right. \\
\Theta_{\mathrm{X}}(R, z)=\left\{\begin{array}{cc}
\tan ^{-1}\left(\frac{|z|}{R-R_{p}}\right) & \left(R \leq R_{\mathrm{X}}^{c}\right) \\
\Theta_{\mathrm{X}}^{0} & \left(R>R_{\mathrm{X}}^{c}\right)
\end{array}\right.
\end{gathered}
$$

and

$$
R_{p}=\left\{\begin{array}{cl}
\frac{R R_{\mathrm{X}}^{c}}{R_{\mathrm{X}}^{c}+|z| / \tan \Theta_{\mathrm{X}}^{0}} & \left(R \leq R_{\mathrm{X}}^{c}\right) \\
R-\frac{|z|}{\tan \Theta_{\mathrm{X}}^{0}} & \left(R>R_{\mathrm{X}}^{c}\right)
\end{array},\right.
$$

where, according to Ref. [21], $B_{\mathrm{X}}^{0}=4.6 \mu \mathrm{G}, \Theta_{\mathrm{X}}^{0}=49^{\circ}, R_{\mathrm{X}}^{c}=4.8 \mathrm{kpc}$, and $R_{\mathrm{X}}=2.9 \mathrm{kpc}$. 

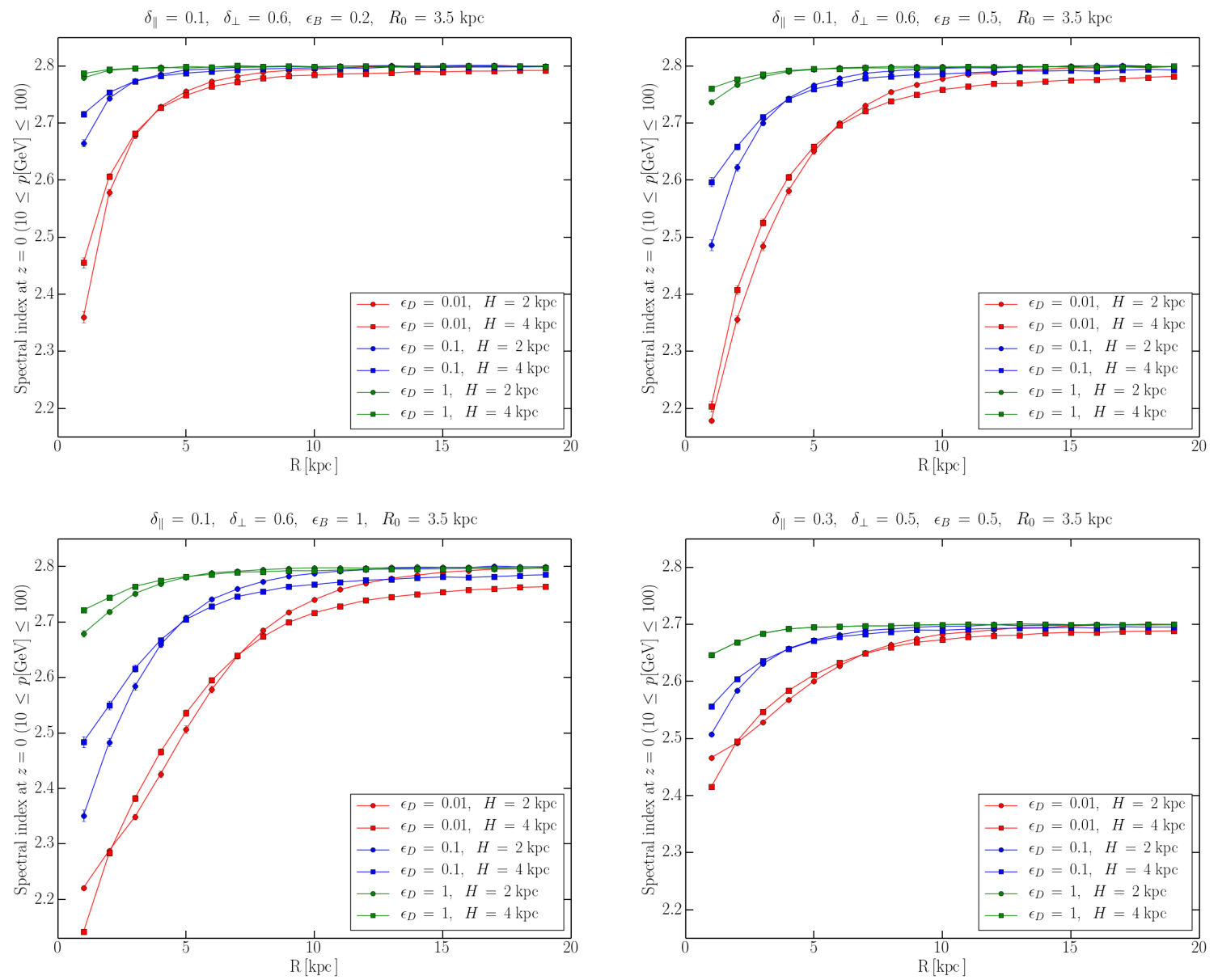

Figure 2: The evolution of the proton spectral index $\alpha$, obtained in the framework of the GMF toy-model defined in Eqs. (2.4)-(2.6), is here shown as a function of the Galactocentric radius $R$. The values of the parameters characterizing the different simulations are reported in the boxed insets and in the title labels.

\section{Results}

We illustrate here the main features of the proton distribution across the Galaxy obtained within the anisotropic propagation setup described in the previous Section. In particular, we start in Section 3.1 with a GMF modelled as in Eqs (2.4)-(2.6) and then, in Section 3.2, we move to the discussion of the realistic case, for which we adopt the GMF described by Eqs. (2.7)-(2.13).

\subsection{Parametric study of the toy model}

The parameters that determine the properties of the GMF toy model are $\varepsilon_{B}$ and $R_{0}$, while the ones that rule the behaviour of parallel and perpendicular diffusion are $\varepsilon_{D}, \delta_{\|}$and $\delta_{\perp}$. We perform a series of simulations with several different sets of values assigned to these parameters in order to better understand their role. The results that we obtain are show in Fig. 2 in terms of the evolution of the proton spectral index $\alpha$ with respect to the Galactocentric radius $R$ across the Galactic plane (i.e. at $z=0$ ). As expected, in all the cases that we consider, $\alpha$ decreases for small values of $R$. 

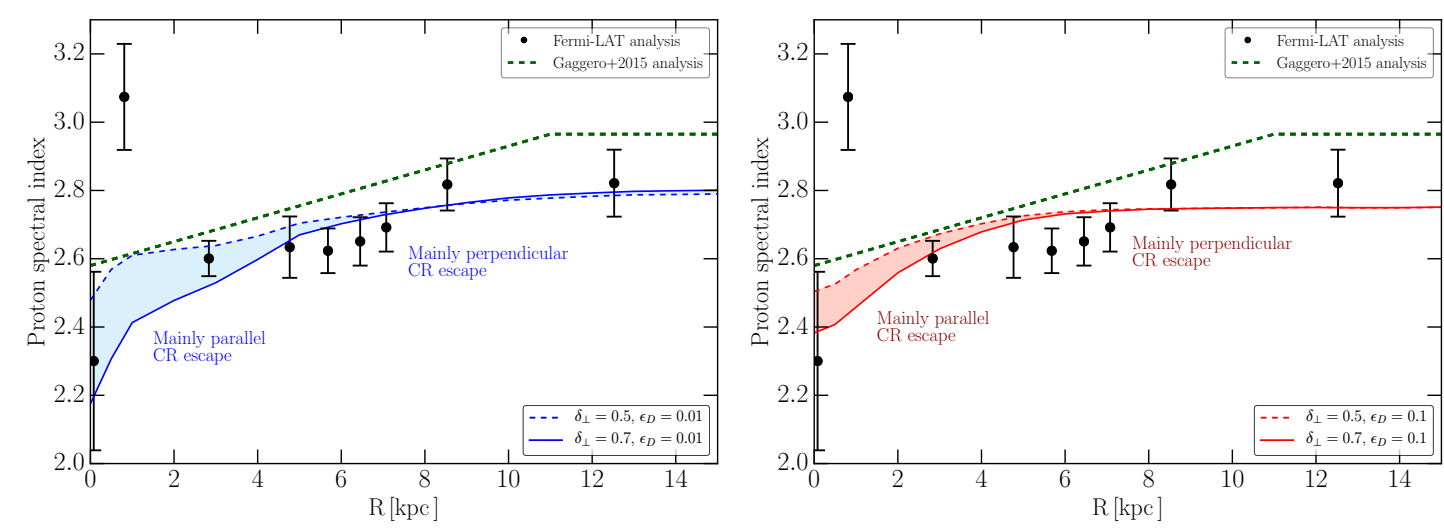

Figure 3: The red lines represent the evolution of the proton spectral index as a function of the Galactocentric radius $R$ for the different setups under consideration. Data points derived from the analysis conducted in [3] are also shown, together with the results obtained by adopting the model proposed in [5] (green dashed line). The left (right) panel refers to the $\varepsilon_{D}=0.01\left(\varepsilon_{D}=0.1\right)$ case.

The reason why this happens is clear once that one considers the definition of the spatial diffusion tensor given in Eq. (2.2), together with the fact that the GMF is predominantly directed along $z$ in the inner Galaxy and increasingly azimuthal for larger values of $R$. In fact, for small values of $R$, protons diffusion is parallel along $z$ and perpendicular along $R$, while at large distances from the Galactic Center it tends to become perpendicular along both directions. More precisely, one can see that, as long as $\delta_{\|}<\delta_{\perp}$, the largest range of variability of $\alpha$ is $\left[\alpha_{\text {inj }}+\delta_{\|}, \alpha_{\text {inj }}+\delta_{\perp}\right]$, the two extremes corresponding, respectively, to the values that $\alpha$ can have at the Galactic Center and at the outer radius of the diffusive halo. It is important to point out that, as it is shown in Fig. 2, such extreme values can be reached only if $\varepsilon_{D} \ll 1$. In fact, if $\varepsilon_{D} \approx 1$, perpendicular transport tends to dominate all across the Galactic plane and the profile of $\alpha$ flattens at $\alpha_{\mathrm{inj}}+\delta_{\perp}$ with just a small hardening at the Galactic Center. The scale of the transition between the extremes values of $\alpha$ is set by the parameter $R_{0}$ that here is fixed to $3.5 \mathrm{kpc}$. The role of the parameter $\varepsilon_{B}$ is similar to the one played by $\varepsilon_{D}$, in the sense that the larger $\varepsilon_{B}$ is the more important parallel transport can be in the inner Galaxy. This can be easily understood if one considers that within this model of the GMF, parallel transport can occur only along the $z$ direction and the magnitude of the diffusion coefficient is proportional to $B_{z}$, which is set by $\varepsilon_{B}$. Another information that one can obtain from Fig. 2 is that the impact of the halo size $H$ on the evolution of $\alpha$ is rather limited and it is again related to the fact that parallel diffusion in this model occurs only along $z$ : a more extended halo can counter-balance the effect of parallel diffusion and allow for a more pronounced effect of perpendicular transport.

\subsection{Realistic model and comparison with Fermi-LAT data}

We fix the values of the parameters ruling the behaviour of the realistic GMF to the values reported in Section 2 and we act only on the parameters related to diffusion, i.e. $\varepsilon_{D}$ and $\delta_{\perp}$. In particular, in light of the parametric study conducted in the above paragraph, we consider those values of $\varepsilon_{D}$ for which the imprint of anisotropic diffusion is more pronounced, i.e. $\varepsilon_{D}=0.1$ and $\varepsilon_{D}=0.01$. We assume $\delta_{\|}=0.3$ we consider values of $\delta_{\perp}$ in the interval $[0.5,0.7]$. 
We show in Fig. 3 the evolution of the proton spectral index with respect to the Galactocentric radius $R$ for the different models under scrutiny, together with the results of the analysis conducted by the Fermi-LAT Collaboration in [3]. Our results are obtained by using for each case under study a different proton injection index in order to match the slope of the local (i.e. at $8.5 \mathrm{kpc}$ ) proton flux as inferred by the Fermi-LAT analysis. As it can be seen, our model predicts a clear hardening towards the Galactic Center. As in the case of the toy-model discussed above, the reason why such feature is realised is that the GMF is predominantly directed along the $z$ direction in the inner region of the Galaxy, while for larger values of the Galactocentric radius $R$ the azimuthal field grows, and so does the impact of perpendicular transport on the spectrum. The transition from the region dominated by parallel transport to the one dominated by perpendicular transport is realised differently, depending on the value of $\varepsilon_{D}$, as it is clear by comparing the two panels of Fig. 3. In addition, the extent of the hardening depends on the value of the $\delta_{\perp}$ parameter. More precisely, the difference in slope between the local spectrum and the spectrum at the Galactic Center is [0.24, $0.36]$ if $\varepsilon_{D}=0.1$ or $[0.27,0.58]$ if $\varepsilon_{D}=0.01$, the lower bound of the interval corresponding to $\delta_{\perp}=0.5$, while the upper one refers to the case where $\delta_{\perp}=0.7$.

Fig. 3 shows also the results obtained by means of the phenomenological model featuring a isotropic but radially-dependent $\delta$ proposed in [5]. Such model appears to be in a good agreement with the results derived here, provided that the proton injection index is opportunely rescaled to match the results of Fermi-LAT analysis.

\section{Conclusions and outlook}

In this work we have presented a two-dimensional numerical solver designed to model CR anisotropic diffusion in the Galaxy. In particular, we have presented the main features of the numerical implementation of the model, we have studied the impact of the modelling of the GMF on the CR distribution at various distances from the Galactic Center, both in the framework of a simplified toy model and within a more realistic scenario. In this last case we have shown how an hardening of the energy distribution of CRs compatible with the hint given by Fermi-LAT data can arise.

This work represents only a first step and further studies are on the way. In particular, a full three-dimensional model of CR transport is needed in order to ascertain the features of the spatial and energetical CR distribution in the Galaxy.

\section{References}

[1] V. L. Ginzburg and S. I. Syrovatskii, The Origin of Cosmic Rays. 1964.

[2] A. W. Strong, I. V. Moskalenko and V. S. Ptuskin, Cosmic-Ray Propagation and Interactions in the Galaxy, Annual Review of Nuclear and Particle Science 57 (Nov., 2007) 285-327, [astro-ph/0701517].

[3] F. Acero et al., Development of the Model of Galactic Interstellar Emission for Standard Point-source Analysis of Fermi Large Area Telescope Data, ApJS 223 (Apr., 2016) 26, [1602.07246].

[4] R.-z. Yang, F. Aharonian and C. Evoli, Radial distribution of the diffuse gamma-ray emissivity in the galactic disk, 1602.04710. 
[5] D. Gaggero, A. Urbano, M. Valli and P. Ullio, Gamma-ray sky points to radial gradients in cosmic-ray transport, Phys. Rev. D91 (2015) 083012, [1411. 7623].

[6] D. Gaggero, D. Grasso, A. Marinelli, A. Urbano and M. Valli, The gamma-ray and neutrino sky: A consistent picture of Fermi-LAT, Milagro, and IceCube results, Astrophys. J. 815 (2015) L25, [1504.00227].

[7] D. Gaggero, D. Grasso, A. Marinelli, M. Taoso and A. Urbano, Diffuse cosmic rays shining in the Galactic center: A novel interpretation of H.E.S.S. and Fermi-LAT gamma-ray data, 1702.01124.

[8] P. Morrison, On the Origins of Cosmic Rays, Reviews of Modern Physics 29 (Apr., 1957) 235-243.

[9] J. R. Jokipii, Cosmic-Ray Propagation. I. Charged Particles in a Random Magnetic Field, ApJ 146 (Nov., 1966) 480.

[10] J. R. Jokipii and E. N. Parker, Random Walk of Magnetic Lines of Force in Astrophysics, Physical Review Letters 21 (July, 1968) 44-47.

[11] D. DeMarco, P. Blasi and T. Stanev, Numerical propagation of high energy cosmic rays in the Galaxy: I. Technical issues, JCAP 6 (June, 2007) 27, [0 705.1972 ].

[12] F. Casse, M. Lemoine and G. Pelletier, Transport of cosmic rays in chaotic magnetic fields, Phy.Rev.D 65 (Jan., 2002) 023002, [astro-ph/0109223].

[13] A. P. Snodin, A. Shukurov, G. R. Sarson, P. J. Bushby and L. F. S. Rodrigues, Global diffusion of cosmic rays in random magnetic fields, MNRAS 457 (Apr., 2016) 3975-3987, [1509.03766].

[14] R. Pakmor, C. Pfrommer, C. M. Simpson and V. Springel, Galactic Winds Driven by Isotropic and Anisotropic Cosmic-Ray Diffusion in Disk Galaxies, ApJL 824 (June, 2016) L30, [1 605.00643 ].

[15] C. Evoli, D. Gaggero, D. Grasso and L. Maccione, Cosmic-Ray Nuclei, Antiprotons and Gamma-rays in the Galaxy: a New Diffusion Model, JCAP 0810 (2008) 018, [0 807.4730 ].

[16] S. S. Cerri, D. Gaggero, A. Vittino, C. Evoli and D. Grasso, A signature of anisotropic cosmic-ray transport in the gamma-ray sky, 1707.07694.

[17] C. Evoli, D. Gaggero, A. Vittino, G. Di Bernardo, M. Di Mauro, A. Ligorini et al., Cosmic-ray propagation with DRAGON2: I. numerical solver and astrophysical ingredients, JCAP 2 (Feb., 2017) 015, [1607.07886].

[18] F. Effenberger, H. Fichtner, K. Scherer and I. Büsching, Anisotropic diffusion of Galactic cosmic ray protons and their steady-state azimuthal distribution, A\&A 547 (Nov., 2012) A120, [1210.1423].

[19] D. R. Lorimer, A. J. Faulkner, A. G. Lyne, R. N. Manchester, M. Kramer, M. A. McLaughlin et al., The Parkes Multibeam Pulsar Survey - VI. Discovery and timing of 142 pulsars and a Galactic population analysis, MNRAS 372 (Oct., 2006) 777-800, [astro-ph/ 0607640 ].

[20] M. S. Pshirkov, P. G. Tinyakov, P. P. Kronberg and K. J. Newton-McGee, Deriving the Global Structure of the Galactic Magnetic Field from Faraday Rotation Measures of Extragalactic Sources, ApJ 738 (Sept., 2011) 192, [1103.0814].

[21] R. Jansson and G. R. Farrar, A New Model of the Galactic Magnetic Field, ApJ 757 (Sept., 2012) 14, [1204.3662]. 